\title{
Liposomes as potential carriers for ketorolac ophthalmic delivery: formulation and stability issues
}

\author{
Mohammed Maher Mehanna ${ }^{1,2^{*}}$, Nouran Abd El-Kader ${ }^{3}$, Magda Wadih Samaha1 \\ ${ }^{1}$ Industrial Pharmacy Department, Faculty of Pharmacy, Alexandria University, Alexandria, Egypt, ${ }^{2}$ Pharmaceutical \\ Technology Department, Faculty of Pharmacy, Beirut Arab University, Beirut, Lebanon, ${ }^{3} R$ \& D Pharco B international \\ Company, Borg El Arab City, Alexandria, Egypt
}

\begin{abstract}
Drug delivery to treat ocular disorders locally is a challenging endeavor. Traditional ocular dosage form - eye drops - exhibits poor availability, consequently inefficient therapeutic response. The objective of the study was to formulate and characterize a ketorolac tromethamine ocular system with a prolonged release pattern based on liposomes as a vesicular carrier and to design once daily liquid preparation realizing the thermal in situ gelation principle. Liposomes were prepared by film hydration method. The influence of cholesterol concentration, $\mathrm{pH}$ and volume of hydration medium, and type and concentration of charging imparting agents were studied. Liposomes were characterized via, morphological examination, vesicular size, and encapsulation efficiency, and in vitro release performance, moreover its stability was assessed. The results obtained highlighted that liposomes showed a closed vesicular multi-lamellar structure. Ketorolac was successfully encapsulated within the liposomal structure in a cholesterol and charge inducing agent concentration-dependent behaviour. The dispersion of liposomes within thermosensitive Poloxamer in situ gel was able to retard the release of the drug by diffusion providing a controlled prolonged delivery. The liposomal formulations were physically stable for six months. Ketorolac tromethamine in situ liposomal gel representing an efficient alternative in terms of ocular retention and patient compliance when compared with conventional eye drops.
\end{abstract}

Uniterms: Ketorolac/characterization/formulation. Liposomes/stability study. Ocular delivery. Thermosensitive gel. Prolonged release.

\section{INTRODUCTION}

Ketorolac tromethamine, Figure 1, is one of the arylacetic acid derivatives group of non-steroidal antiinflammatory drugs which are potent cyclooxygenase inhibitors (Ahuja et al., 2008). It has been used in the management of several ocular disorders. Ketorolac is FDA approved treatment of ocular pain following corneal refractive surgery and surface ablation and other ocular conditions such as seasonal allergic conjunctivitis and postoperative inflammation (Robinson, Lee, 2011). Ketorolac, free acid, is sparingly soluble in water and, therefore, it is marketed in the form of tromethamine salt, which has a higher aqueous solubility (Thakur, Kashiv, 2011). Ketorolac tromethamine is commercially available

\footnotetext{
*Correspondence: M. M. Mehanna. Industrial Pharmacy Department. Faculty of Pharmacy. Alexandria University, Egypt. E-mail: mmhanna@bau.edu.le, dr_mehanna@yahoo.com
}

as eye drops. Instillation of $0.5 \%$ ketorolac tromethamine aqueous solution was associated with ocular irritation, evoked as burning and stinging (Sandoval et al., 2006).

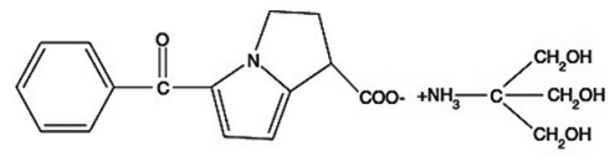

FIGURE 1 - Chemical structure of ketorolac tromethamine.

The main problem encounter eye drops as a local ocular drug delivery is its poor bioavailability due to short residence time on the eye surface as a result of several factors among them, preparation overflow from the eye surface specially upon blinking, dilution by tears, reflex lachrymation and systemic drug absorption through nasolacrimal drainage (Snell, 1998). In order to prevail over these limitations, numerous trials were carried out to prolong the contact time between the preparation and 
the ocular surface such as ketorolac oily solution and ophthalmic ointment preparations had been studied and evaluated (Malhotra, Majumdar, 2006). Gelling systems were utilized for the same purpose as ion activated in situ gel (Vodithala et al., 2010), hydrogel system based on chitosan and carbopol 940 (Zaki et al., 2011), methylcellulose and hydroxypropyl methylcellulose in presence of sodium bicarbonate (Nanjawade, Manvi, Manjappa, 2007). Recently, Thakor et al. (2012), formulated ketorolac tromethamine in various in-situ gelling systems, which significantly improved ocular bioavailability as compared to conventional eye drops as proved through in vitro and in vivo studies through its longer precorneal residence time and ability to sustain drug release.

Phospholipid-based vesicles, i.e. liposomes, are biocompatible and biodegradable carrier system with almost no cytotoxicity. Moreover, it has the capability of enhancing drug solubility, stability and reduce drug adverse reactions (Schubert, 2002). From ophthalmic formulation point of view, liposomes are able to intimately contact the corneal and conjunctival surfaces and modify the tears dynamics which positively reflect on its ability to prolong the drug ocular availability and thus reducing the frequency of administration, the total drug applied and both the systemic and topical side effects which augment patient compliance to the drug regimen. On top of these merits, liposomal preparations are still a liquid formulation that are easily installed with no discomfort and can localize and maintain drug activity at the ocular surface for a longer period, thus allowing a controlled drug delivery (Van Der Bijl, Van Eyk, Meyer, 2001). Several research clusters investigated the utilization of liposomes as ocular system exemplified by the study of Mehanna, Elmaradny and Samaha (2009) who formulated ciprofloxacin loaded-liposomes using the reverse phase evaporation technique and studied five different factors affecting the entrapment efficiency; the results revealed that the most important factor was the molar concentration of cholesterol, Habi, Fouad, Fathalla (2008) prepared $2 \mathrm{mg} / \mathrm{mL}$ fluconazole loadedliposomes using reverse phases evaporation technique for the treatment of Candida keratitis in rabbits eyes and found that the liposomal formulation showed a better healing percentage over the same period of time. In 2014, Tahaa et al. (2014) studied several liposomal formulations containing ciprofloxacin and examined the influence of different types of phospholipids, cholesterol incorporation, incorporation of positively charge inducing agent, and ultrasonication on the liposomes properties and concluded that liposomal formulations showed more than three folds of improvement in drug ocular bioavailability compared with the commercial product.

The goal of the present study is to prepare, characterize and optimize an ocular liposomal formulation of ketorolac tromethamine as a potent anti-inflammatory drug for treatment of ocular conditions with a prolonged release. Furthermore, formulate the prepared liposomes in a thermo-sensitive gel. The physical stability of the optimized liposomal formulation along with the liposomal gel is evaluated upon storage at different temperatures.

\section{MATERIAL AND METHODS}

\section{Material}

Ketorolac tromethamine (assay $<99.0 \%$ ), Dicetyl phosphate, Stearylamine and Pluronic F 127(PF-127) were purchased from Sigma Aldrich (Steinheim, Switzerland). Lipoid E80, phosphatidylcholine from egg was received as a gift from Lipoid Company (Ludwigshafen, Germany). Cholesterol was purchased from Winlab (Leicestershire, UK). Spectrapore ${ }^{\circledR} 2$ dialysis membrane form Spectrum laboratories Inc. (Houston, TX, USA). All other solvents and materials used were of analytical grade.

\section{Preparation of Ketorolac tromethamine-loaded liposomes}

Liposomes were prepared using film hydration technique, which involves dissolving the organic soluble moieties (phospholipid, cholesterol and charge inducing agent) in an organic solvent, then evaporating this solvent by means of vacuum and gentle heat, leaving an even film on the walls of the round-bottom flask. The second major step is the 'hydration' that is carried out with an aqueous phase composed of water, drug and other water-soluble ingredients. This hydration causes immediate globulization of the phospholipid film where the lipid layer tends to form liposomes incorporating the aqueous phase either inside the bilayer of the liposomal wall and/or in the core of the lipid vesicle. The final step is the refrigeration of the formed liposomes to ensure intact vesicle formation.

Specifically, $100 \mathrm{mg}$ phosphatidyl choline dissolved in chloroform, along with cholesterol, and other organic soluble ingredients. The flask is held in rotavapor under approximately $-900 \mathrm{mbar}, 50{ }^{\circ} \mathrm{C}$, rotating at $45 \mathrm{rpm}$ (Rotavapor, type V8000, Buchi, Switzerland) till the solvent is completely evaporated and an even film is formed. Hydration is achieved by addition of the aqueous phase including the ketorolac tromethamine (KT) 
under normal pressure at $50{ }^{\circ} \mathrm{C}$, rotating at $45 \mathrm{rpm}$, for 20 minutes till the film is completely hydrated and the liposomes are formed, then stored in refrigerator for 24 hours (Ghanbarzadeh, Valizadeh, Zakeri-Milani, 2013) a $3\left(2 .^{2}\right.$

\section{Separation of unentraped free ketorolac tromethamine}

Free ketorolac tromethamine separation from liposomal dispersion was performed using cooling ultracentrifuge (Sigma laboratory refrigerated centrifuge, $3 \mathrm{~K}-30$, Germany) rotating at $17000 \mathrm{rpm}$, at a temperature of $1^{\circ} \mathrm{C}$, for 90 minutes. The supernatant representing the unentraped ketorolac tromethamine was decanted then transferred to a volumetric flask suitably diluted and drug content was determined spectrophotometrically (Shimadzu UV 1601 PC spectrophotometer, Kyoto, Japan). Liposomes was redispersed with $1 \mathrm{~mL}$ distilled water and stored for further characterization (Mehanna, Elmaradny, Samaha, 2010).

\section{Optimization of ketorolac liposomal entrapment efficiency}

In order to develop an optimized liposomal formulation with the highest entrapment efficiency, many variables have been explored namely; the molar ratio of cholesterol to that of the phospholipid, various $\mathrm{pH}$ value of hydration medium selected based on the $\mathrm{pk}_{\mathrm{a}}$ of the drug, varying the aqueous hydration phase volume, incorporation of charge inducing agent and finally finding out the concentration of the charge imparting agent. These experiments were assessed by comparing the entrapment efficiency percentage. Table I illustrates the composition of different liposomes dispersions.

\section{Physicochemical characterization of ketorolac- loaded liposomes}

\section{Determination of entrapment efficiency (EE\%)}

Entrapment efficiency was calculated from the difference between the initial drug added during preparation and the free drug determined in the supernatant and expressed as percent entrapment efficiency, which is defined as the percent fraction of the total input drug encapsulated in the lipid bilayers and/or aqueous compartments in the liposome structure (Mehanna, Elmaradny, Samaha, 2009). Entrapment efficiency percentage was computed from the following equation:

$\%$ Entrapment efficiency $=\frac{\text { initial concentration }- \text { concentration of unentraped KT }}{\text { initial concentration }} * 100$
Morphological examination of KT-loaded liposomes

Ketorolac tromethamine-loaded liposomes were morphologically inspected using optical microscope (Euromed, Holland) in order to justify their formation and identify their shapes. Liposome dispersions were observed under a binocular microscope fitted with a camera. A drop of liposomes dispersion was placed on a microscope slide (magnification power 2000), then viewed and photographed.

\section{Transmission electron microscope examination (TEM)}

Ketorolac-loaded liposomal dispersion was examined by TEM (JEM-100S; Joel, Tokyo, Japan); negatively stained sample was prepared by applying a drop of liposomal dispersion to copper-coated grids, after 2 min, the excess was drawn off with filter paper. A saturated uranyl acetate aqueous solution was used as a staining agent. The excess was eliminated with distilled water and the samples were analyzed by transmission electron microscope at magnification power 10,000 at $80 \mathrm{KV}$.

\section{Vesicular size analysis}

Liposomes size was assessed using laser diffractometer (CilasL100, model 1064 liquid; Quantachrom, France), Determining the mean vesicle size and size distribution (polydispersity) by calculating the span index, assures the uniformity of the liposomal preparation, calculated using the following equation:

$$
\text { Span index }=D_{v, 90}-D_{v, 10} / D_{v, 50}
$$

where $\mathrm{D}_{(\mathrm{v}, 10)} \mathrm{D}_{(\mathrm{v}, 50)}$ and $\mathrm{D}_{(\mathrm{v}, 90)}$ are the equivalent volume diameters at 10, 50 and $90 \%$ cumulative volumes, respectively (Mehanna, Elmaradny, Samaha, 2014).

\section{In-vitro release studies}

The release of ketorolac tromethamine from the different liposomal dispersions was determined using dialysis apparatus method (Ma et al., 2008). Fixed volume of each formula in a $5 \mathrm{~cm}$ semipermeable dialysis membrane (Molecular weight cutoff 12,000-14,000 Da) tied from both sides and clamped to the paddle of the dissolution instrument (DT820, Erweka, Germany) rotating at $50 \mathrm{rpm}$, temperature kept at $34.5 \pm 1{ }^{\circ} \mathrm{C}$ (simulating eye temperature), immersed in $250 \mathrm{ml}$ phosphate buffer saline (PBS) as a releasing medium, samples were withdrawn at different time intervals and directly compensated with fresh PBS kept at the same temperature. The drug concentrations in the withdrawn samples were measured spectrophotometrically using PBS as blank at $\lambda \max 324 \mathrm{~nm}$. 
TABLE I - Composition of ketorolac tromethamine -loaded liposomes dispersions*

\begin{tabular}{lcccccc}
\hline Formula & $\begin{array}{c}\text { Phospholipids } \\
(\mathbf{m g})\end{array}$ & $\begin{array}{c}\text { Cholesterol } \\
(\mathbf{m g})\end{array}$ & $\mathbf{p H}$ & $\begin{array}{c}\text { Hydration } \\
\text { volume (mL) }\end{array}$ & $\begin{array}{c}\text { Dicetyl } \\
\text { phosphate } \\
\text { (mmol) }\end{array}$ & $\begin{array}{c}\text { Stearyl amine } \\
(\mathbf{m m o l})\end{array}$ \\
\hline F.1 & 100 & $\mathbf{0}$ & 7.4 & 5 & -- & -- \\
F.2 & 100 & $\mathbf{2 0}$ & 7.4 & 5 & -- & -- \\
F.3 & 100 & $\mathbf{3 3}$ & 7.4 & 5 & -- & -- \\
F.4 & 100 & $\mathbf{5 0}$ & 7.4 & 5 & -- & -- \\
F.5 & 100 & 50 & $\mathbf{3}$ & 5 & -- & -- \\
F.6 & 100 & 50 & $\mathbf{4 . 2}$ & $\mathbf{5}$ & -- & - \\
F.7 & 100 & 50 & 7.4 & 5 & -- & - \\
F.8 & 100 & 50 & 4.2 & $\mathbf{2 . 5}$ & -- & -- \\
F.9 & 100 & 50 & 4.2 & $\mathbf{1 0}$ & -- & -- \\
F.10 & 100 & 50 & 4.2 & 5 & $\mathbf{0 . 5}$ & -- \\
F.11 & 100 & 50 & 4.2 & 5 & -- & $\mathbf{0 . 5}$ \\
F.12 & 100 & 50 & 4.2 & 2.5 & -- & $\mathbf{0 . 1}$ \\
F.13 & 100 & 50 & 4.2 & 2.5 & -- & $\mathbf{0 . 5}$ \\
F.14 & 100 & 50 & 4.2 & 2.5 & -- & $\mathbf{1}$ \\
\hline
\end{tabular}

* All formulation contain $1 \%$ ketorolac tromethamine

Release kinetics of Ketorolac from the prepared liposomal dispersions, were examined based on the magnitude of correlation coefficients obtained after application of zero order, first order, and Higuchi diffusion, Peppeas-Korsmeyer and Hixon Crowell models (Dash et al., 2010).

\section{Preparation of thermosensitive liposomal gel}

Pluronic F127 polymer undergoes sol-to-gel phase transition upon exposure to physiological eye temperature - was practically found to have a gelling concentration of $22 \%$, was employed as a vehicle for liposomes. The gel preparation was performed according the cold method (Mehanna, Elmaradny, Samaha, 2013); where PF-127 is accurately weighed and slowly added to cold water $\left(5^{\circ} \mathrm{C}\right)$ with constant stirring, then refrigerated for 5 hours for complete polymer hydration and get rid of air bubbles which resulted from stirring. Liposomal PF-127 gel formula was prepared through dispersing KT-loaded liposomes in the PF-127 solutions under magnetic stirring in ice.

\section{Physical stability of liposomes}

The physical stability of KT-loaded liposomes was assessed via monitoring the liposomes size and secondly, by chemical quantitation of entrapment efficiency percentage through different time intervals for six months at room temperature $25{ }^{\circ} \mathrm{C}$ and in refrigerator at $4{ }^{\circ} \mathrm{C}$. In addition, stability of liposomes incorporated in thermosensitive gel was assessed through the same time intervals by chemical quantitation of entrapment efficiency percent.

\section{RESULTS AND DISCUSSION}

\section{Optimization of ketorolac loaded-liposomal entrapment efficiency}

Ketorolac tromethamine encapsulated within liposomes with a simple film hydration method was performed aiming to prolong the retention time of the drug within the eye hence enhancing its therapeutic antiinflammatory effect. In order to optimize the liposomes capacity to entrapment $\mathrm{KT}$, several variables were studied as shown in table I. The factors investigated were; inclusion of cholesterol and its molar concentration, hydration at three different $\mathrm{pH}$ values, namely; $\mathrm{pH} 3,4.2$, and 7.4, hydration volume, and the incorporation of charge inducing agents, dicetyl phosphate as a negative charge inducing agent and stearylamine as a positive charge inducing agent. The effect of varying the concentration of the charge-inducing agent was also addressed. The entrapment efficiency percentage was selected as the determining governing response. 


\section{Effect of molar cholesterol concentration}

Ketorolac tomethamine loading level in liposomes prepared by film hydration method was expressed as entrapment efficiency percentage (EE\%), which was varied between 15 to $19 \%$, based on the molar concentration of cholesterol included within the liposomes (F1-F4). This low entrapment efficiency can be explained as a result of drug high water solubility (Hou et al., 1990).

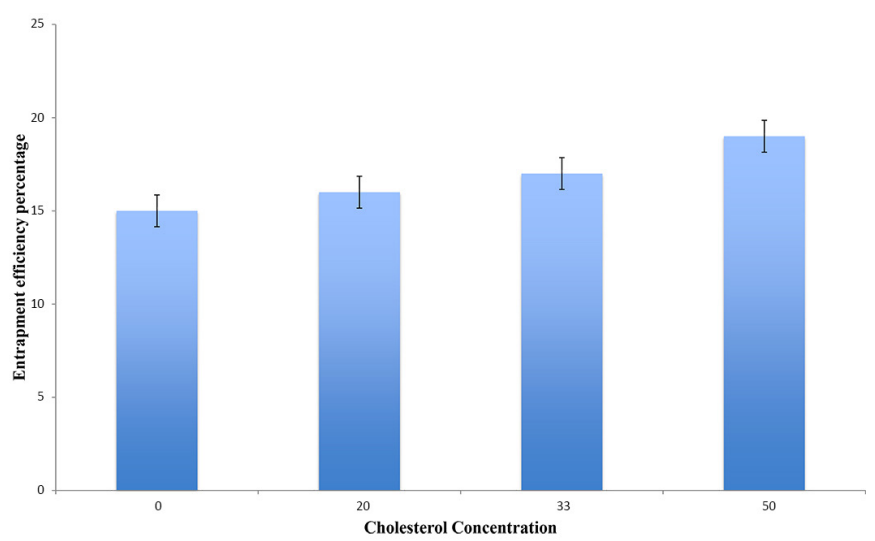

FIGURE 2 - Entrapment efficiency of ketorolac-loaded liposomes with various cholesterol molar concentration.

The positive correlation between the molar concentration of cholesterol and EE\% could be infer from Figure 2. This could be attributed to the hardening of the liposomal walls due to increase cholesterol concentration. Cholesterol molecules augment the structure integrity of liposomes bilayers membrane as it fills the spaces between the phospholipid molecules, in turns it reduces the membrane permeability and drug leakage, thus enhancing drug loading which was in accordance to many previously reported data ( Mehanna, Elmaradny, Samaha, 2010; Hosny, 2010; Alsarra et al., 2005). Correspondingly, liposomes contain 50\% molar cholesterol percentage was selected for further optimization.

\section{Effect of hydration phase $\mathrm{pH}$ and volume}

In order to study the influence of different $\mathrm{pH}$ values of the aqueous phase containing KT, Formulas 5, 6 and 7 corresponding to $\mathrm{pH} 3,4.2$ and 7.4 were tested. Ketorolac tromethamine precipitated in $\mathrm{pH} 3$ buffer during the preparation F5 and thus it was not possible to use it as a hydrating solution during the early preparation stages. This precipitation can be seen as a result of poor drug solubility in $\mathrm{pH} 3$, which is below its $\mathrm{pKa}$ (3.5) that stopped the experiment early. Although the $\mathrm{pH} 7.4$ is similar to the ocular physiological $\mathrm{pH}$ minimizing irritation and discomfort effect, still liposomes hydrated with ketorolac tromethamine in buffer $\mathrm{pH} 4.2$ gave a higher $\mathrm{EE} \%$ i.e.
$57 \%$. This $\mathrm{pH}$ is the same for the marked FDA approved product. Similar results were observed by Mehanna, Elmaradny and Samaha (2009). Moreover, Malhotra and Majumdar (2002) found that ketorolac tromethamine is more permeable in goats' corneas at acidic $\mathrm{pH}$ than $\mathrm{pH}$ 7.5.

Moreover, the volume of aqueous phase was varied 2.5-10 $\mathrm{mL}$ and its effect on ketorolac tromethamine entrapment was assessed, the highest EE\% was that obtained with the smallest hydration volume, $2.5 \mathrm{~mL}$, as shown in Figure 3. During the practical work, the least volume was observed to give a white dense colloidal liquid of high consistency, probably the reason for more efficient entrapment. These results are supported by the high water solubility of KT, which favor the aqueous medium leading to a reduction in $\mathrm{EE} \%$ upon increasing the hydration volume.

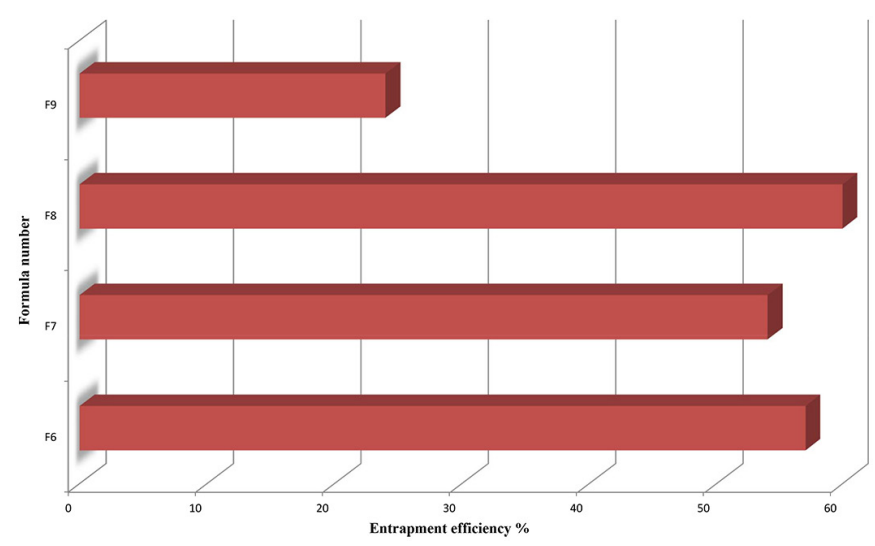

FIGURE 3 - Effect of hydration phase $\mathrm{pH}$ and volume on the entrapment efficiency of ketorolac-loaded liposomes.

\section{Effect of incorporation and concentration of charge imparting agents}

Upon comparing neutrally charged liposomal formula (F6) to negatively charge liposomal formula (F10), and to positively charge liposomal formula (F11) by incorporation of dicetyl phosphate and stearylamine, respectively, it was noted that the positively charged liposomes had the highest EE\% compared to other liposomes. Meanwhile, the negatively charged and the neutral liposomes showed lower entrapment efficiencies (Table II). An explanation to this observation is KT is negatively charged at $\mathrm{pH} 4.2$ thus electrostatic attraction to the positive charged stearyl amine preventing ketorolac tromethamine from leaking outside the liposome and enhance its $\mathrm{EE} \%$ in addition to the electrostatic repulsion between the multiple bilayers of the liposomes inducing an elevation of the aqueous phase inclusion within the liposomal bilayers and indirectly positevly influence KT entrapment. The higher EE\% of the negatively charged 
TABLE II - Effect of incorporation and concentration of charge imparting agents on ketorolac tromethamine -loaded liposomes entrapment efficiency

\begin{tabular}{lcccc}
\hline \multirow{2}{*}{ Formula } & \multicolumn{2}{c}{ Charge imparting agents concentration (mMole) } & & $\begin{array}{c}\text { Entrapment } \\
\text { efficiency } \%\end{array}$ \\
\cline { 2 - 3 } & \multicolumn{2}{c}{ Dicetyl phosphate } & Stearylamine & \\
\hline F10 & 0.5 & - & 38 \\
F11 & - & 0.5 & 41 \\
F12 & - & 0.1 & 37 \\
F13 & - & 0.5 & 63 \\
F14 & - & 1 & 58 \\
\hline
\end{tabular}

*Formula prepared with $5 \mathrm{ml}$ aqueous hydration volume

liposomes compared to neutral vesicles is based on solely on the repulsion between bilayers. Similar results were obtained by Schaeffer and Krohn (Schaeffer, Krohn 1982). Whereas, Hosny (2010) has found that the entrapment efficiency was best in positively charged liposomes, while the neutral liposomes were better than the negatively charged liposomes this might be due to the zwitter ion nature of ciprofloxacin drug.

To compare the effect of varying the concentration of positive charge imparting agent (stearylamine) on KT liposomes entrapment efficiency, three formulations were prepared and tested using $0.1,0.5$ and 1.0 mmole (F12, F13 and F14, respectively. Through table 2, it could be observed that increasing the molar concentration of stearylamine was linked to increase in $\mathrm{EE} \%$ up to 0.5 mmole but further increase led to a reduction of KT entrapment. As higher stearylamine concentration may lead to disruption of liposomes structure and formation as it lacks the amphiphilic nature of phospholipids. These results are quite similar to Mehanna, Elmaradny and Samaha (2009) who reported that 0.38 molar ratio of stearylamine was the optimal for ciprofloxacin-loaded liposomes preparation.

\section{Morphological examination of ketorolac tromethamine-loaded liposomes}

The microscopical examination using the optical microscope -magnifying at 2000x- showed well-identified spherical vesicular structure with large internal space of the liposomes as illustrated in Figure 4, liposomes without cholesterol appeared as transparent bi-layer, whereas liposomes containing cholesterol showed a fortified bilayers, It is agreed by many researchers that the incorporation of cholesterol increases the rigidity of the bilayer membranes (Tahaa et al., 2014; Wessman, Edwards, Mahlin, 2010). The transmission electron microscope magnifying at $10,000 x$ showed mainly multilamellar liposomes with some unilamellar vesicles as evident in Figure 4. Several authors reported comparable findings (Sinico et al., 2005).
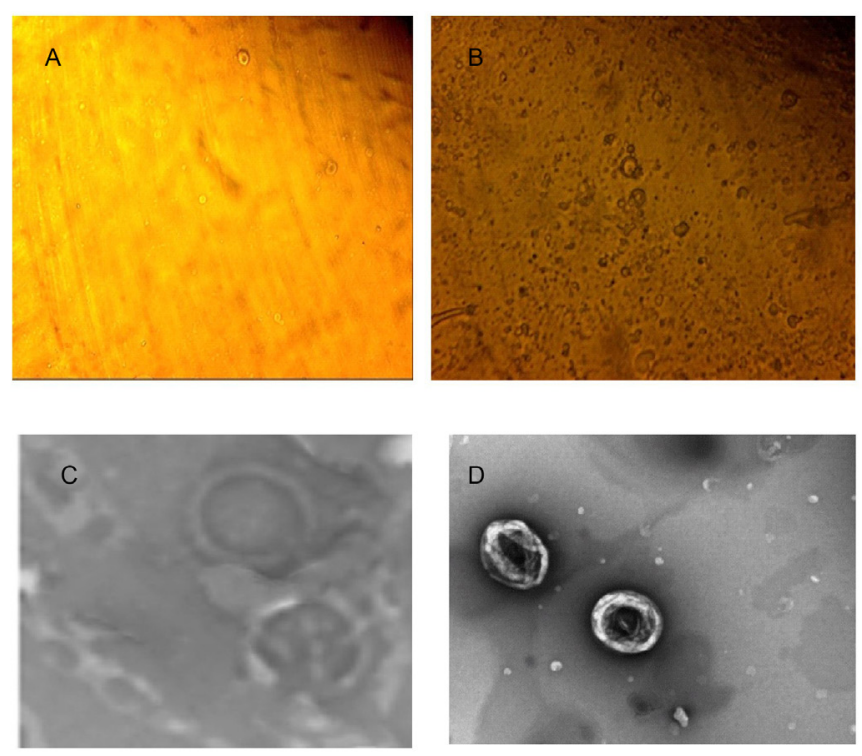

FIGURE 4 - Optical photographs (A and B) and transmission electron microscopical photomicrographs (C and D) of Ketorolac tromethamine-loaded liposomes prepared via film hydration method.

\section{Particle size evaluation of ketorolac tromethamine-loaded liposomes}

The average size and size distribution are important parameters as to determine the mechanism of uptake by the cornea, as well their stability condition of liposomes (Gaudana et al., 2010). The particle size of nanoliposomes was investigated using a particle size analyzer (Zeta Sizer 2000, Malvern Instruments, Worcestershire, UK), as well as transmission electron microscope that elaborated liposomes size range between 196-8350 nm, varying according to the formula of liposomes. The span index 
indicates the uniformity of the liposomal preparation, the smaller the span index, and the narrower the size distribution.

As evident by the results illustrated in Table III, a dramatic increase in the particle size of the liposomes upon increasing the cholesterol concentration (F1F4). Increasing the molar cholesterol concentration reflected on a significant increase in the overall size of the liposomes. The amphiphilic nature of cholesterol controls its organization within the liposomal bilayer membrane structure as inserts itself into the bilayer with its hydrophilic head oriented towards the aqueous surface and aliphatic chain line up parallel to the hydrocarbon chains in the center of the bilayers which induced the observed enlargement of liposomes (Xu, London, 2000). These results were previously reported by Tahaa et al. (2014) who found that liposomes size increased linearly with increasing the cholesterol concentration. Similar effect of cholesterol inclusion was reported for nevirapine (Ramana et al., 2010).

TABLE III - Particle size and size distribution indicated by Span Index of ketorolac tromethamine-loaded liposomes

\begin{tabular}{lcc}
\hline Formula & Particle size (nm) & Span Index \\
\hline F1 & 196 & 0.064 \\
F2 & 3270 & 0.960 \\
F3 & 7000 & 0.540 \\
F4 & 7050 & 0.390 \\
F10 & 8350 & 0.450 \\
F11 & 7060 & 0.430 \\
F13 & 2900 & 0.950 \\
\hline
\end{tabular}

It is also obvious that charged liposomes (F10 \& F11) had a larger particle size when compared to the neutral liposomes with the same initial components (F1-F4). This behaviour can be linked to the electrostatic repulsion between the charged molecules, which led to expand the spaces between the bilayers due to pushing the polar heads of the phospholipids outwards within the multilamellar structure (Hosny, 2010; Ramana et al., 2010). A more closed examination of the results elaborated the influence of the nature of the charging inducing agent as the positively charged liposomes were of higher particle size than the negatively charged ones. This could be attributed to the bulkiness of dicetyl phosphate (C32 H67 O4 P) molecule with its two cetyl chains might have resulted in less tightly packed bilayer membranes compared to the less bulky stearylamine (C8 H15 N O8), molecule. Another possible explanation is the charge density that would amplify the electrostatic repulsion force between the dicetyl phosphate and cholesterol head groups having a similar charge (Samad, Sultana, Aqil, 2007). Therefore, the overall result showed that the positively charged liposomes (F11) were smaller than the negatively charged ones (F10) i.e. uncharged liposomes $<$ positively charged $<$ negatively charged liposomes.

Therefore, it could be deduced that increasing the concentration of cholesterol resulted in an increase in the liposomes size consequently increased the entrapment efficiency. Thus, the particle size is directly proportional to the entrapment efficiency of liposomes.

\section{In-vitro release studies}

In order to develop a liposomal drug delivery system for localized and sustained ocular delivery, it was necessary to check the release profile of different dispersions in conditions simulating the ocular environment. Since human tears are known to be complex, approximately isotonic solution containing proteins, lipids, electrolytes and other components with $\mathrm{pH}$ values in the range of 6.8-7.5 (Abelson, Udell, Weston, 1980). The principle-buffering agent in the eye is bicarbonate system, which is unstable overtime when used for laboratory studies. The simulated tear solution used was isotonic phosphate buffer ( $\mathrm{pH} 7.4)$, which was chosen for its $\mathrm{pH}$ stability, analytical non-interference and relationship to human tear $(\mathrm{pH}$, buffering range, and buffering capacity). Furthermore it was desired to keep the simulated tear solution simple so as to prevent complications from possible interactions and analytical interference (Carney, Mauger, Hill, 1989).

The release profile of ketorolac from the optimized liposomal preparation (F13) was illustrated in Figure 5. Upon comparing the release profile of the optimized formula of ketorolac-loaded liposomes to the marketed product, it was found that the release profile of ketorolacloaded liposomes showed the slowest release profile with initial burst release. This performance may be contributed to the desorption of the electrically attached drug molecules on liposomes outer surface followed by slower release rate of the encapsulated drug within the multilamellar liposomal structure (Widjaja et al., 2014).

With the intention of eliminating the burst release and controlling ketorolac release from liposomes. PF127-based liposomal gel was prepared and evaluated. This strategy was proved to be able to control the drug release over $24 \mathrm{~h}$ as shown in Figure 5, which illustrates the cumulative percent of ketorolac released as a function 


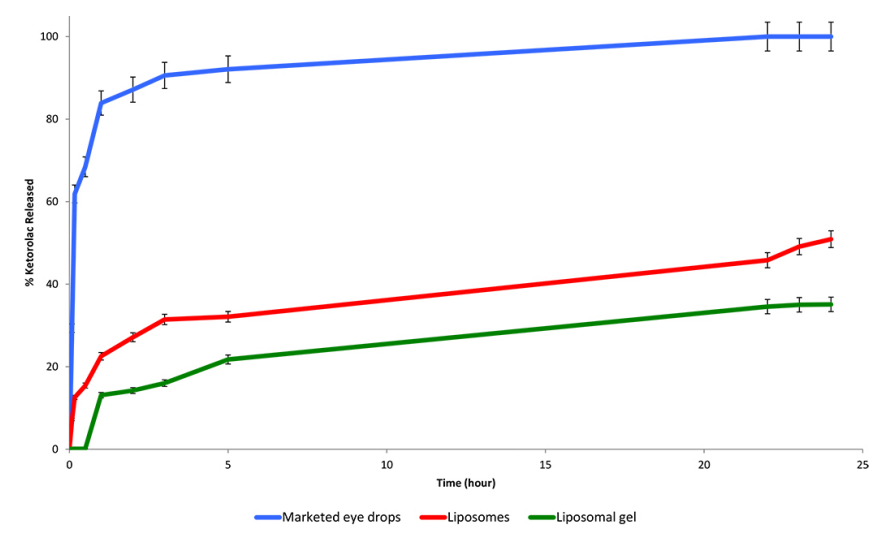

FIGURE 5 - Release profile of ketorolac tromethamine into phosphate buffer saline at $34.5 \pm 1^{\circ} \mathrm{C}$ from marketed eye drops, liposomes dispersion and thermosensitive liposomal gel.

of time. The controlled release manner was mainly due to gel nature of PF- 127 at $34.5 \pm 1{ }^{\circ} \mathrm{C}$, which increase the viscosity of the diffusion layer surrounding the liposomes representing another barrier for drug release. The explanation is further supported by the kinetic analysis, which indicated that ketorolac release mechanism was a diffusion-dependent process as the best fit for kinetic models was Higuchi model based on the correlation coefficient. Comparable results were reported by several authors (Nagarsenker, Londhe, Nadkarni, 1999; Jain, Shastri, 2011).

\section{Stability of liposomal formulation}

A stability study for six months was performed to assess the ability of the prepared liposomes to maintain its physicochemical characteristics. The optimized liposomal formula (F13), and the in-situ liposomal thermoresponsive gel were stored at 4 and $25^{\circ} \mathrm{C}$. The physical appearance of the liposomes formulations seemed unchanged, neither sedimentation nor flocculation was observed indicating the high physical stability of the liposomal system in general. The results obtained are tabulated (Table IV) showing that liposomal formula (F13), stored at room temperature $\left(25^{\circ} \mathrm{C}\right)$ showed a faster decrease in $\mathrm{EE} \%$ than that of refrigerated liposomes $\left(4{ }^{\circ} \mathrm{C}\right)$ that indicate the leakage of the entrapped drug from the vesicular structure. The elevated temperature induced energy-dependent fusion of liposomes via enhancing its Brownian motion in addition; the phospholipid became more flexible and fluid through increased temperature, thus the entrapped drug leaked easily. Comparable outcomes were obtained by Manosroi and Podjanasoothon (2002) who studied the effect of temperature on different tranexamic acid liposomal formulations proving that the high temperature led to the highest leakage of drug. Moreover, liposomal in-situ gelling system was able to maintain drug encapsulation at both temperatures compared to the liposomes dispersion. A more stable formulation was that stored at $25^{\circ} \mathrm{C}$, which might be due to the thermosensitive gel formation at elevated temperature. One can conclude that, liposomes in gel showed better retaining of the drug i.e. better $\mathrm{EE} \%$ as a two barrier systems not allowing the drug to leak out of the liposomes.

TABLE IV - Influence of storage temperature on the entrapment efficacy of ketorolac tromethamine-loaded liposomes

\begin{tabular}{lccc}
\hline \multirow{2}{*}{ Formula } & \multirow{2}{*}{$\begin{array}{c}\text { Time interval } \\
\text { (Month) }\end{array}$} & \multicolumn{2}{c}{$\begin{array}{c}\text { Entrapment } \\
\text { efficiency \% }\end{array}$} \\
\cline { 3 - 4 } & & $\mathbf{4}^{\circ} \mathbf{C}$ & $\mathbf{2 5}^{\circ} \mathbf{C}$ \\
\hline Liposomal aqueous & 1 & 96 & 93 \\
dispersion & 3 & 92 & 87 \\
& 6 & 85 & 75 \\
\hline Liposomal & 1 & 98 & 99 \\
thermoresponsive & 3 & 92 & 95 \\
gel & 6 & 88 & 92 \\
\hline
\end{tabular}

\section{CONCLUSION}

In the current study, the potential of liposomes as ketorolac vesicular carrier for ocular delivery was explored. A controlled release ocular dosage form based on ketorolac tromethamine liposomes was successfully formulated, optimized and characterized. It has been shown that liposomes prepared using fifty percent cholesterol, with low hydrating volume, at $\mathrm{pH} 4.2$, and using a positive charge-imparting agent at $0.5 \mathrm{mM}$ concentration, resulted in the highest entrapment efficiency which is the main key for hydrophilic drug liposomal encapsulation. In addition, It was found that the mechanism of release from the liposomes to the eye was diffusion. Liposomes dispersed in Pluronic F-127 in situ thermosensitive gel demonstrated a prolonged release over $24 \mathrm{~h}$ with more sustainability of the dose due to the dual barrier which the drug has to penetrate. The stability study of liposomes and liposomal gel at 4 and $25^{\circ} \mathrm{C}$ for six months, showed that the liposomal gel at room temperature $\left(25^{\circ} \mathrm{C}\right)$ had the best stability. Ketorolac tromethamine-loaded liposomal thermoresponsive-based gel representing a practical stable substituent to the ordinary eye drops via its capability to control and prolong the drug release with reduce frequency of dosing and in reflect augment patient compliance which is a major problem in ocular therapy. 


\section{REFERENCES}

ABELSON, M.B.; UDELL, I.J.; WESTON, J.H. Normal human tear $\mathrm{pH}$ by direct measurement. Arch. Ophthalmol. Chic., v.99, n.2, p.301-304, 1980.

AHUJA, M.; DHAKE, A.S.; SHARMA, S.K.; MAJUMDAR, D.K. Topical ocular delivery of NSAIDs. AAPS J., v.10, n.2, p.229-241, 2008.

ALSARRA, I.A.; BOSELA, A.A.; AHMED, S.M.; MAHROUS, G.M. Proniosomes as a drug carrier for transdermal delivery of ketorolac. Eur. J. Pharm. Biopharm., v.59, n.3, p.485490, 2005.

CARNEY, L.G.; MAUGER, T.F.; HILL, R.M. Buffering in human tears: $\mathrm{pH}$ responses to acid and base challenge. Invest. Ophth. Vis. Sci., v.30, n.4, p.747-754, 1989.

DASH, S.; MURTHY, P.N.; NATH, L.; CHOWDHURY, P. Kinetic modeling on drug release from controlled drug delivery systems. Acta. Pol. Pharm., v.67, n.3, p.217-23, 2010.

GAUDANA, R.; ANANTHULA, H.K.; PARENKY, A.; MITRA, A.K. Ocular drug delivery. AAPS J., v.12, n.3, p.348-360, 2010.

GHANBARZADEH, S.; VALIZADEH, H.; ZAKERI-MILANI, P. Application of response surface methodology in development of sirolimus liposomes prepared by thin film hydration technique. Bioimpacts, v.3, n.2, p.75-81, 2013.

HABIB, F.S.; FOUAD, E.A.; FATHALLA, D. Liposomes as an ocular delivery system of fluconazole: In-vitro studies. Bull. Pharm. Sci., v.31, pt.2, p.293-311, 2008.

HOSNY, K.M. Ciprofloxacin as ocular liposomal hydrogel. AAPS Pharmscitech., v.11, n.1, p.241-246, 2010.

HOU, X.P.; CUI, D.H.; YI, Y.Y.; LI, X.Y.; YANG, L.P. Hydrophobic modification of water soluble drug and its reconstitutable liposomes. Acta Pharm. Sinic., v.25, n.11, p.854-858, 1990.

JAIN, R.; SHASTRI, J. Study of ocular drug delivery system using drug-loaded liposomes. Int. J. Pharm. Invest., v.1, n.1, p.35, 2011.
MA, W. D.; XU, H.; WANG, C.; NIE, S.F.; PAN, W.S. Pluronic F127-g-poly(acrylic acid) copolymers as in situ gelling vehicle for ophthalmic drug delivery system. Int. J. Pharm., v.350, n.1-2, p.247-256, 2008.

MALHOTRA, M.; MAJUMDAR, D.K. Aqueous, oil, and ointment formulations of ketorolac: efficacy against prostaglandin E2-induced ocular inflammation and safety: a technical note. AAPS Pharmscitech., v.7, n.4, p.96, 2006.

MALHOTRA, M.; MAJUMDAR, D.K. Effect of preservative, antioxidant and viscolizing agents on in vitro transcorneal permeation of ketorolac tromethamine. Indian J. Exp. Biol., v.40, n.5, p.555-559, 2002.

MANOSROI, A.; PODJANASOONTHON, K. Stability and release of topical tranexamic acid liposome formulations. J. Cosmet. Sci., v.386, p.375-386, 2002.

MEHANNA, M.M.; ELMARADNY, H.A.; SAMAHA, M.W. Ciprofloxacin liposomes as vesicular reservoirs for ocular delivery: formulation, optimization, and in vitro characterization. Drug Dev. Ind. Pharm., v.35, n.5, p.583593, 2009.

MEHANNA, M.M.; ELMARADNY, H.A.; SAMAHA, M.W. Mucoadhesive liposomes as ocular delivery system: physical, microbiological, and in vivo assessment. Drug Dev. Ind. Pharm., v.36, n.1, p.108-118, 2010.

MEHANNA, M.M.; ELMARADNY, H.A.; SAMAHA, M.W. Thermosensitive liposomal gel as an ocular delivery system: physical, microbiological and in vivo assessments. Pharm. Ind., v.75, n.4, p.681-689, 2013.

MEHANNA, M.M.; ELMARADNY, H.A.; SAMAHA, M.W. Nanovesicular carrier-mediated transdermal delivery of tadalafil: i-formulation and physicsochemical characterization. Drug Dev. Ind. Pharm., v.41, n.5, p.714721, 2014.

NAGARSENKER, M.S.; LONDHE, V.Y.; NADKARNI, G.D. Preparation and evaluation of liposomal formulations of tropicamide for ocular delivery. Int. J. Pharm., v.190, n.1, p.63-71, 1999.

NANJAWADE, B.K.; MANVI, F.V.; MANJAPPA, A.S. In situforming hydrogels for sustained ophthalmic drug delivery. J. Control. Release, v.122, n.2, p.119-134, 2007. 
RAMANA, L.N.; SETHURAMAN, S., RANGA, U.; KRISHNAN, M.U. Development of a liposomal nanodelivery system for nevirapine. J. Biomed. Sci., v.17, p.57, 2010.

ROBINSON, J.R.; LEE, V.H. Controlled drug delivery: fundamentals and applications. 2.ed. New York: Informa Health Care, 2011. 744p.

SAMAD, A.; SULTANA, Y.; AQIL, M. Liposomal drug delivery systems: an update review. Curr. Drug Deliv., v.4, n.4, p.297-305, 2007.

SANDOVAL, H.P.; CASTRO, L.E.F.; VROMAN, D.T.; SOLOMON, K.D. Evaluation of $0.4 \%$ ketorolac tromethamine ophthalmic solution versus $0.5 \%$ ketorolac tromethamine ophthalmic solution after phacoemulsification and intraocular lens implantation. J. Ocul. Pharmacol. Ther., v.22, n.4, p.251-257, 2006.

SCHAEFFER, H.E.; KROHN, D.L. Liposomes in topical drug delivery. Invest. Ophth. Vis. Sci., v.22, n.2, p.220-227, 1982.

SCHUBERT, R. Medical applications of liposomes. Eur. J. Pharm. Biopharm., v.54, n.3, p.358-359, 2002.

SINICO, C.; MANCONI, M.; PEPPI, M.; LAI, F.; VALENTI, D.; FADDA, A.M. Liposomes as carriers for dermal delivery of tretinoin: in vitro evaluation of drug permeation and vesicle-skin interaction. J. Control. Release, v.103, n.1, p.123-136, 2005.

SNELL, S.R.; LEMP, M.A. Clinical anatomy of the eye. 2.ed. Oxford: Wiley-Blackwell, 1998. 400p.

TAHAA, E.I.; EL-ANAZIA, M.H.; El-BAGORYB, I.M.; BAYOMIA, A.M. Design of liposomal colloidal systems for ocular delivery of ciprofloxacin. Saudi. Pharm. J., v.22, n.3, p.231-239, 2014.
THAKOR, S.; VHORA, I.; DESAI, J.; THAKKAR, S.; THAKKAR, H. Physiologically activated phase transition systems for improved ocular retention of ketorolac tromethamine. J. Pharm. Bioal. Sci., v.4, n.1, p.s6-s7, 2012.

THAKUR, R.R.; KASHIV, M. Modern delivery systems for ocular drug formulations: a comparative overview W.R.T conventional dosage form. Int. J. Res. Pharm. Biomed. Sci., v.2, n.1, p.8-18, 2011.

VAN DER BIJL, P.; VAN EYK, A.D.; MEYER, D. Effects of three penetration enhancers on transcorneal permeation of cyclosporine. Cornea, v.20, n.5, p.505-508, 2001.

VODITHALA, S.; KHATRY, S.; SHASTRI, N.; SADANANDAM, M. Formulation and evaluation of ion activated ocular gels of ketorolac tromethamine. Int. J. Curr. Pharm. Res., v.2, n.3, p.33-38, 2010.

WESSMAN, P.; EDWARDS, K.; MAHLIN, D. Structural effects caused by spray- and freeze-drying of liposomes and bilayer disks. J. Pharm. Sci., v.99, n.4, p.2032-2048, 2010.

WIDJAJA, L.K.; BORA, M.; CHAN, P.N.; LIPIK, V.; WONG, T.T.; VENKATRAMAN, S.S. Hyaluronic acidbased nanocomposite hydrogels for ocular drug delivery applications. J. Biomed. Mater. Res. A, v.102, n.9, p.3056$3065,2014$.

XU, X.; LONDON, E. The effect of sterol structure on membrane lipid domains reveals how cholesterol can induce lipid domain formation. Biochem., v.39, n.5, p.843-849, 2000 .

ZAKI, R.; HOSNY, M.K.; MOHAMED, A.K.; ABD-ELBARY, A. Ketorolac tromethamine in-situ ocular hydro gel; preparation, characterization, and in-vivo evaluation. Int. J. Pharm., v.3, n.3, p.535-545, 2011.

Received for publication on $29^{\text {th }}$ June 2016 Accepted for publication on $20^{\text {th }}$ January 2017 